\title{
Higher Risks of Virologic Failure and All-Cause Deaths Among Older People Living with HIV in Chongqing, China
}

\author{
Guohui Wu, ${ }^{1}$ Chao Zhou, ${ }^{1}$ Xiangjun Zhang, ${ }^{2}$ Wei Zhang,, Rongrong Lu, Lin Ouyang, \\ Hui Xing, ${ }^{3}$ Yiming Shao, ${ }^{3}$ Yuhua Ruan, ${ }^{3}$ and Han-Zhu Qian ${ }^{4,5}$
}

\begin{abstract}
Older people living with HIV (PLWH) may have delayed diagnosis and access to care and therefore have poorer disease outcomes. Little is known about HIV care and disease outcomes among older PLWH in China. This retrospective cohort study used data from all adult HIV/AIDS cases during 1988-2017 in Chongqing, China from two national databases. We compared demographic and behavioral profiles, HIV care, virologic suppression, and mortality between two age groups of 18-49 and $\geq 50$ years. Multivariate logistic and cox regression analyses were used to calculate adjusted odds ratio (AOR) and adjusted hazard ratio (AHR) among older versus younger PLWH. Of 46,580 adult HIV/AIDS cases, $76.1 \%$ were men and $38.2 \%$ were 50 years of age or older. The proportion of older cases in men increased from $2.4 \%$ in 2002 to $51.8 \%$ in 2017, and in women from $3.3 \%$ to $57.9 \%$. Older PLWH had a lower CD4 count than their younger counterparts at HIV diagnosis (median 323 vs. 391 cells $/ \mu \mathrm{L} ; p<.001$ ). The average time from HIV diagnosis to initiation of antiretroviral therapy (ART) were 6.3 months among older and 12.8 months among younger PLWH $(p<.001)$. Nearly one tenth $(9.6 \%)$ had virologic failure within 12 months of ART initiation, and the odds of virologic failure among older PLWH was $80 \%$ higher [AOR 1.8; 95\% confidence interval (CI), 1.1-3.0] than among younger ones after controlling for calendar year of initiating ART and other covariates. The mortality rate within 12 months of initiating ART was 9.8 deaths per 100 person years, and the risk of mortality among older PLWH was three times among younger ones (AHR, 3.1; 95\% CI, 2.1-4.6). Older people represented an increasing proportion of new HIV/AIDS cases and were more likely to have virologic failure and mortality within 12 months of ART initiation.
\end{abstract}

Keywords: HIV, older people, antiretroviral therapy, virologic failure, all-cause mortality, China

\section{Introduction}

$\mathbf{H}^{\mathrm{v}}$ IV PREVALENCE AMONG people 50 years of age and older has been increasing steadily in the past two decades globally. ${ }^{1,2}$ The increase in the proportion of older individuals among people living with HIV (PLWH) is due primarily to the successes of highly active antiretroviral therapy (ART), which has prevented early deaths and extended the lives of individuals infected at a younger age. ${ }^{3} \mathrm{~A}$ smaller but increasing share of older adult cases are attributable to new infections in later life. ${ }^{4-6}$

Older PLWH may have different demographic and sociobehavioral profiles and clinical and immunological conditions, which affect outcomes of the disease. ${ }^{7-11}$ Both patients and clinicians often underestimate the risk of HIV among older adults, and this low-risk perception may cause delays in HIV testing and diagnosis. ${ }^{12-14}$ In addition, older PLWH face more challenges across stages of HIV care

\footnotetext{
${ }^{1}$ Chongqing Municipal Center for Disease Control and Prevention, Chongqing, China.

${ }^{2}$ School of Community Health Sciences, University of Nevada, Reno, Reno, Nevada.

${ }^{3}$ State Key Laboratory of Infectious Disease Prevention and Control (SKLID), Chinese Center for Disease Control and Prevention (China CDC), Collaborative Innovation Center for Diagnosis and Treatment of Infectious Diseases, Beijing, China.

${ }^{4}$ Shanghai Jiao Tong University-Yale Joint Center for Biostatistics and Data Science, School of Life Sciences and Biotechnology, Shanghai Jiao Tong University, Shanghai, China.

${ }^{5}$ Department of Biostatistics, Yale School of Public Health, New Haven, Connecticut.

(c) Guohui Wu et al. 2019; Published by Mary Ann Liebert, Inc. This Open Access article is distributed under the terms of the Creative Commons License (http://creativecommons.org/licenses/by/4.0), which permits unrestricted use, distribution, and reproduction in any medium, provided the original work is properly cited.
} 
continuum due to later diagnosis and ART initiation in the disease course, ${ }^{15,16}$ a faster decline in immune function, ${ }^{17}$ and higher mortality rates, ${ }^{6}$ compared with younger people. For example, older age at ART initiation was associated with increased mortality in a large cohort of ART initiators in Latin America and the Caribbean. ${ }^{9}$

Current literature on HIV epidemic and care among older people is mainly from non-Asian countries. HIV epidemic in China is unique in some aspect. For example, it was initially driven by injecting drug use and contaminated plasma donation in the 1980s and 1990s, and since then it has transformed as heterosexual and homosexual transmissions. One notable feature of recent epidemic is high risk of homosexual transmission in young men, accounting for nearly $60 \%$ reported cases among the age group of 15-24 years in 2017. Another feature is rapid increase among men older than 60 years, with reported cases from 4,751 in 2010 to 19,815 in 2017 (unpublished data from China CDC). Yet few studies have investigated HIV issues among older Chinese people. A prospective cohort study among rural residents in southwestern China found that HIV incidence was 2.7 per 10,000 person years in all participants, but 71.3 among a subpopulation of males 50-69 years of age who had less than secondary schooling and were divorced or widowed. ${ }^{5}$ Another Chinese study found that older HIV-infected adults reported a lower level of wellbeing, higher level of depression, and poorer quality of life. ${ }^{18} \mathrm{~A}$ qualitative study found that older males had prevalent unsafe sex, and both male and female older adults had low awareness of HIV risk. ${ }^{19}$ To better understand the disease characteristics among older PLWH in China, we performed a retrospective cohort analysis of HIV epidemic, disease status, and access to care among all reported HIV/AIDS cases in Chongqing City, China.

\section{Methods}

\section{Study site}

Chongqing is the largest municipality that is directly administered by the central government of China and plays a key role in the development strategy for western China. It includes 25 districts and 13 counties, and has a total population of 30 million, including about 8.5 urban registered residents.

The first HIV infection was diagnosed in Chongqing in 1988, 3 years later than the first reporting of HIV/AIDS cases in 1985 in China. Between 2007 and 2012, the reported HIV infections grew at an average annual rate of $19.7 \%$, substantially higher than the national rate $(3.1 \%) .^{20}$

\section{Data sources}

Data were extracted from China's two web-based national databases for real-time collection and maintenance of information related to HIV case reporting and treatment, respectively. All newly identified HIV/AIDS cases from local hospitals, CDC clinics, and blood banks are reported through the HIV/AIDS case reporting system (CIS) at regular intervals, which includes demographic information and the date and venue of HIV diagnosis and the date and value of first CD4 test. The first CD4 test was typically performed immediately after HIV diagnosis. The HIV treatment system, called the HIV/AIDS Comprehensive Response Information Management System (CRIMS), has been described else- where, ${ }^{21}$ which includes information on ART regimens, viral load, comorbid diseases, loss to follow-up, transfer to other region, and death. Patients who initiated ART are followed at 15 days, 1 and 3 months, and then every 3 months, and their information on ART is updated in CIIMS accordingly. The recommended schedule for viral testing within 12 months of ART initiation is once every 1-2 months, but many patients do not follow the schedule. Designated staff at Chongqing Municipal Center for Disease Control and Prevention (Chongqing CDC), who have access to the records of HIVinfected individuals who are the residents of Chongqing City, downloaded the deidentified data from the online databases.

The study period covered from January 1, 1988 when the first case was reported in Chongqing City, to December 31, 2017. Because screening for inclusion was across the city and included all identified adult cases 18 years of age or older, sample size was not initially defined.

Variables used in this study included date of birth, date of HIV diagnosis, self-reported sexual orientation/most probable sources of HIV transmission, gender, death, CD4 count from CIS, HIV viral load, ART, and death and comorbidities from CRIMS. The inclusion criteria were: (1) registered resident in Chongqing; (2) 18 years of age or older; and (3) HIV-positive reported during January 1, 1988 and December 31,2017 . The data are anonymous and delinked from individual identifiable information.

\section{Ethics statement}

This study utilizes anonymous data and data from secondary sources, which do not contain any personal identifiers. Therefore, ethics approval was not needed.

\section{Data analysis}

The age of each participant was measured at the date of HIV diagnosis, which is also the time point of entering the cohort. Older people are defined as HIV-infected adults who were 50 years or older at HIV diagnosis. ${ }^{22}$ We calculated the proportions of older PLWH in all adult cases in each calendar year by sex.

We compared the demographic and behavioral characteristics between older (age 250 ) and younger PLWH (ages 1849). We also compared HIV care between two age groups in men and women, respectively. Chi-square tests were used for categorical variables and t-tests were used for continuous variables.

We evaluated the difference of HIV treatment outcomes by age group, including virologic failure and mortality within 12 months of initiating ART. Considering the 6-month window of viral load testing, we assessed virologic failure up to 15 months after ART initiation for each participant. Multivariate logistic regression analysis was performed to calculate adjusted odds ratio and $95 \%$ confidence interval (CI) of virologic failure within 12 months of initiating ART among older age group while adjusting for potential confounders, including HIV transmission route and calendar year of initiating ART. Only those who had started ART and had viral load data within 12 months of ART initiation were included in this analysis. Viral load data were extracted from CRIMS until October 31, 2018. As older group had a higher proportion of heterosexual transmissions, the younger group had a higher proportion of homosexual transmissions, and an interaction 
term of age group and transmission route was included in the model. Cox regression analysis was performed to assess the relationship between older age and all-cause deaths within 12 months of initiating ART while adjusting for HIV transmission route and other covariates. Only those who had started ART and had information on survival status within 12 months of ART initiation were included in this analysis. As Chinese free ART programs started standardized treatment in 2008, one individual who started ART before 2008 was excluded from the analysis. Censorship was at the earliest recorded date of death, loss to follow-up, transfer out of the cohort, or up to 12 months of initiating ART. All analyses were performed in SPSS version 19.0.

\section{Results}

Trend of HIV epidemic among older adults in Chongqing

During years 1988-2017, a total of 46,580 adult HIV/AIDS cases were reported in Chongqing, and $76 \%$ were men and $24 \%$ were women (Table 1). Of all adult PLWH, $38.2 \%(n=17,815)$ were 50 years of age or older. The average proportion of older cases in men was $37.2 \%(13,186 /$ 35,428 ) during 1988-2017, increasing from $2.4 \%$ in 2002 to $51.8 \%$ in 2017 . The proportion of older cases in women was $41.5 \%(4,629 / 11,152)$ on average, increasing from $3.3 \%$ in 2002 to $57.9 \%$ in 2017 (Fig. 1).

Table 1. Comparison of Demographic and Behavioral Characteristics Among HiV-Infected Individuals Between Ages 18-49 and $\geq 50$ Years in Chongqing, China, 1988-2017

\begin{tabular}{|c|c|c|c|c|}
\hline & Total, N (\%) & Ages 18-49, N (\%) & Age $\geq 50, \mathrm{~N}(\%)$ & $\mathrm{p}$ \\
\hline Total & $46,580(100)$ & $28,765(61.8)$ & $17,815(38.2)$ & \\
\hline Sex & & & & $<.001$ \\
\hline Male & $35,428(76.1)$ & $22,242(77.3)$ & $13,186(74.0)$ & \\
\hline Female & $11,152(23.9)$ & $6,523(22.7)$ & $4,629(26.0)$ & \\
\hline Ethnicity & & & & $<.001$ \\
\hline Han & $45,258(97.2)$ & $27,749(96.5)$ & $17,509(98.3)$ & \\
\hline Other & $1,322(2.8)$ & $1,016(3.5)$ & $306(1.7)$ & \\
\hline Marital status & & & & $<.001$ \\
\hline Single & $12,690(27.2)$ & $11,750(40.9)$ & $940(5.3)$ & \\
\hline Currently married & $22,982(49.3)$ & $11,866(41.3)$ & $11,116(62.4)$ & \\
\hline Divorced or separated or widowed or other & $10,113(21.7)$ & $4,532(15.8)$ & $5,581(31.3)$ & \\
\hline Unknown & $795(1.8)$ & $617(2.1)$ & $178(1.0)$ & \\
\hline Education & & & & $<.001$ \\
\hline Junior middle school or lower & $32,352(69.5)$ & $16,576(57.6)$ & $15,776(88.6)$ & \\
\hline Senior high school & $7,589(16.3)$ & $6,025(21.0)$ & $1,564(8.8)$ & \\
\hline College and above & $6,378(13.7)$ & $5,924(20.6)$ & $454(2.5)$ & \\
\hline Unknown & $261(0.5)$ & $240(0.8)$ & $21(0.1)$ & \\
\hline Employment & & & & $<.001$ \\
\hline Employed & $25,114(53.9)$ & $14,174(49.3)$ & $10,940(61.4)$ & \\
\hline Unemployed/retired & $14,092(30.3)$ & $8,622(30.0)$ & $5,470(30.7)$ & \\
\hline Student & $1,026(2.2)$ & $1,026(3.6)$ & $0(0.0)$ & \\
\hline Other & $6,348(13.6)$ & $4,943(17.2)$ & $1,405(7.9)$ & \\
\hline Place of birth & & & & $<.001$ \\
\hline Large city & $17,652(37.9)$ & $12,043(41.9)$ & $5,609(31.5)$ & \\
\hline Medium city & $18,945(40.7)$ & $10,526(36.6)$ & $8,419(47.3)$ & \\
\hline Small city & $7,228(15.5)$ & $4,088(14.2)$ & $3,140(17.6)$ & \\
\hline Township/countryside & $2,450(5.3)$ & $1,816(6.3)$ & $634(3.6)$ & \\
\hline Unknown & $305(0.7)$ & $292(1.0)$ & $13(0.1)$ & \\
\hline Legal Chongqing Residency & & & & $<.001$ \\
\hline Yes & $43,866(94.2)$ & $26,432(91.9)$ & $17,434(97.9)$ & \\
\hline No & $2,714(5.8)$ & $2,333(8.1)$ & $381(2.1)$ & \\
\hline Route of HIV infection & & & & $<.001$ \\
\hline Injecting drug use & $3,404(7.3)$ & $3,187(11.1)$ & $217(1.2)$ & \\
\hline Heterosexual contact & $33,026(70.9)$ & $16,471(57.3)$ & $16,555(92.9)$ & \\
\hline Homosexual contact & $9,055(19.4)$ & $8,285(28.8)$ & $770(4.3)$ & \\
\hline Other & $1,095(2.4)$ & $822(2.9)$ & $273(1.5)$ & \\
\hline Calendar year of HIV diagnosis & & & & $<.001$ \\
\hline 1988-2003 (Pre ART) & $195(0.4)$ & $188(0.7)$ & $7(0.1)$ & \\
\hline 2004-2007 (No standardized ART) & $2,571(5.5)$ & $23,69(8.2)$ & $202(1.1)$ & \\
\hline 2008-2012 (Standardized ART) & $12,293(26.4)$ & $9,008(31.3)$ & $3,285(18.4)$ & \\
\hline 2013-2017 (TDF added) & $31,521(67.7)$ & $17,200(59.8)$ & $14,321(80.4)$ & \\
\hline
\end{tabular}

ART, antiretroviral therapy; TDF, tenofovir disoproxil fumarate. 


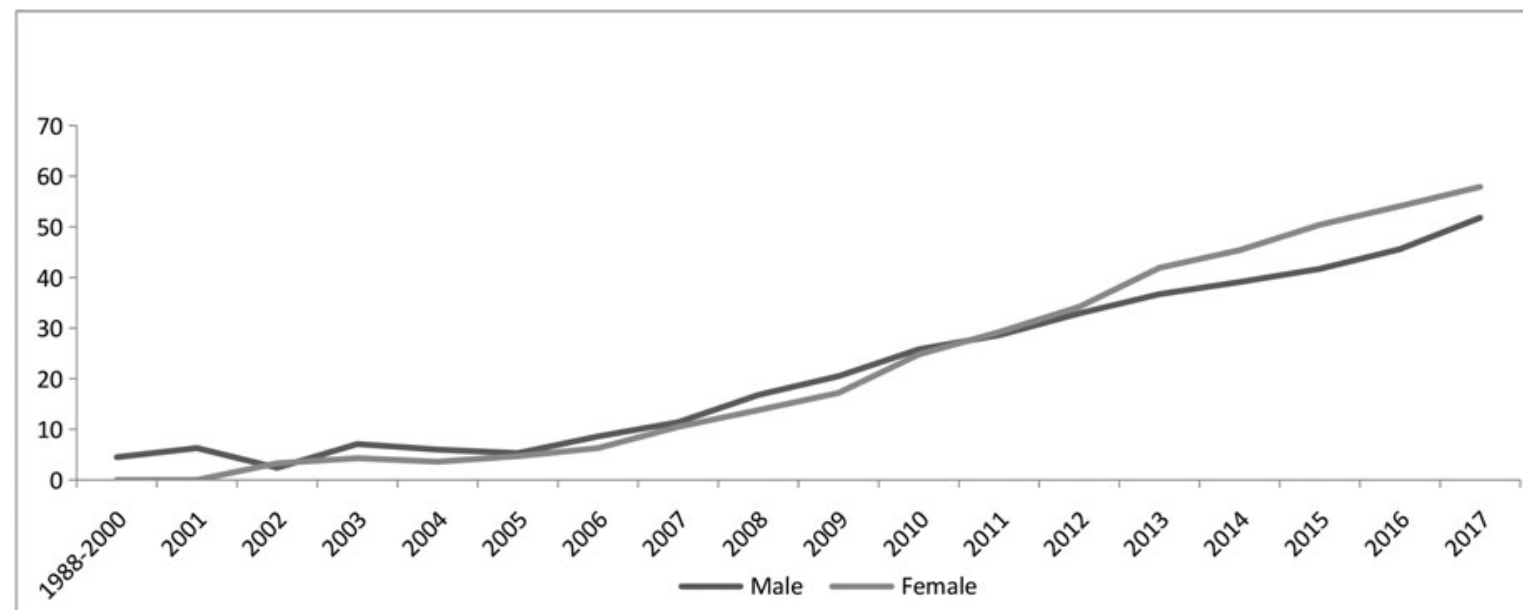

\begin{tabular}{|l|c|c|c|c|c|c|c|c|c|c|c|c|c|c|c|c|c|c|}
\hline Year & $\begin{array}{c}1988- \\
2000\end{array}$ & 2001 & 2002 & 2003 & 2004 & 2005 & 2006 & 2007 & 2008 & 2009 & 2010 & 2011 & 2012 & 2013 & 2014 & 2015 & 2016 & 2017 \\
\hline Female & 15 & 7 & 30 & 23 & 83 & 129 & 190 & 220 & 340 & 507 & 525 & 699 & 912 & 1101 & 1404 & 1457 & 1670 & 1840 \\
\hline Male & 22 & 16 & 41 & 42 & 168 & 681 & 486 & 614 & 936 & 1581 & 1602 & 2272 & 2919 & 3734 & 4399 & 4828 & 5320 & 5767 \\
\hline
\end{tabular}

FIG. 1. Proportion $(\%)$ of older ( $\geq 50$ years) versus younger (18-49 years) HIV/AIDS cases by sex in Chongqing, China, 1988-2017. The table at the bottom of figure shows the number of new HIV/AIDS cases by sex and calendar year.

\section{Demographic characteristics among older PLWH}

The range of age was 18-49 and 50-91 in two age groups, and the mean (standard deviation) was $35.2(8.8)$ and 62.0 (8.1), respectively. Table 1 compares the demographic and behavioral characteristics between two age groups. Most cases were ethnically Han for both older (98.3\%) and younger groups $(96.5 \%)$. Only $5 \%$ older but $40 \%$ younger PLWH were single, and older PLWH (31.3\%) were more likely to be divorced or widowed than younger people $(15.8 \%, p<.001)$. Older PLWH had a lower education level and a higher proportion of full-time employment than younger people. Compared with younger PLWH, older people were more likely to report heterosexual contact as HIV transmission route $(92.9 \%$ vs. $57.3 \%, p<.001)$, and were less likely to report homosexual risk $(4.3 \%$ vs. $28.8 \%, p<.001)$.

\section{HIV care among older PLWH}

Chinese people can obtain free voluntary HIV testing at CDC clinics, and they may also get paid HIV testing in hospitals when they do medical exams or surgical operations. Less frequently, they can get a test through other ways, for example, buying testing kits online or at drug stores for selftesting. Of reported HIV/AIDS cases in Chongqing, the majority were diagnosed in hospital or CDC, and older cases were more likely to be diagnosed in hospital than younger cases $(68.3 \%$ vs. $44 \%)$; this pattern of age group difference presents in both men and women. Older people had a lower CD4 count [median 323 cells $/ \mu \mathrm{L}$, interquartile range 196429] than younger people (391, 264-501), and this pattern also presents in both men and women. About two third $(67.8 \%)$ of adult PLWH were on ART, and there was no difference between older and younger men (69.9\% vs. $65.8 \%$, $p>.05)$, but there was a slightly higher proportion among older women $(72.3 \%)$ than younger women $(67.8 \%, p<.05)$.
The average time from HIV diagnosis to ART initiation were 6.3 months among older PLWH, which was about half for younger adults $(12.8$ months, $p<.001)$; this difference was similar across all categories of CD4 count and in both men and women (Table 2).

\section{Comparison of virologic failure between older and younger $P L W H$}

As Chinese free ART program did not standardize until 2008, we included participants who started ART since 2008 in this analysis. Of 25,693 participants who had started ART during 2008-2017 and were followed up within 12 months of initiating ART, 11,549 (45\%) had information on viral load, including $46 \%(8,992 / 16,662)$ in younger and $43 \%(5,152 /$ $9,031)$ in older age groups. Of those having viral load data, nearly one tenth $(9.6 \%)$ had virologic failure within 12 months of initiating ART, and this proportion was higher among older $(11.9 \%)$ compared with younger people $(8.5 \%$, $(p<.05)$. The odds of virologic failure for older PLWH was $80 \%$ higher (adjusted odd ratio, 1.8; 95\% CI, 1.1-3.0) than younger ones after controlling for calendar year of initiating ART, HIV transmission route, and interaction term of age group by transmission route (Table 3).

\section{Comparison of mortality between older and younger age groups}

Among 29,241 adult PLWH who started ART and had data on survival status within 12 months of initiating ART, 2,732 deaths were observed in this time period, and the overall mortality rate was 9.8 deaths per 100 person years. Older PLWH had about twice mortality rate compared with younger ones $(14.7 \%$ vs. $6.8 \%)$. The risk of mortality for older PLWH was three times among younger ones (adjusted hazard ratio, 3.1; 95\% CI, 2.1-4.6) after controlling for calendar year 


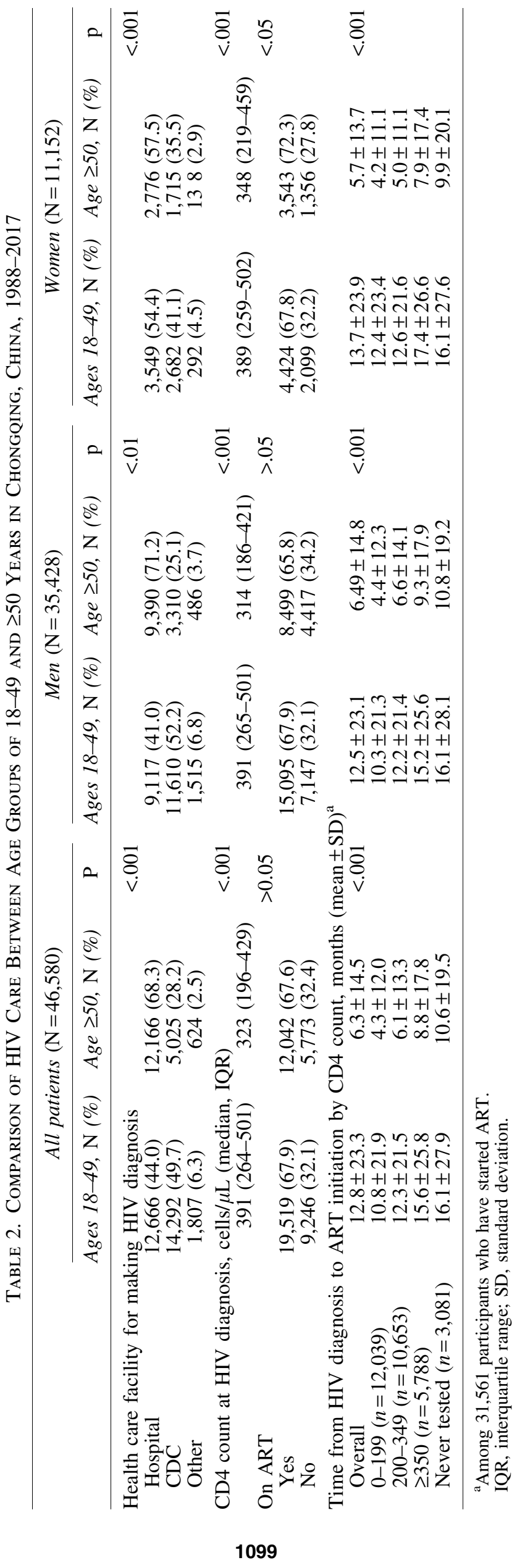


Table 3. Comparison of HIV Virologic Failure Between Age Groups of $18-49$ and $\geq 50$ Years Within 12 (Range 9-15) Months of Initiating Antiretroviral Therapy in Chongqing, China, 2008-2017

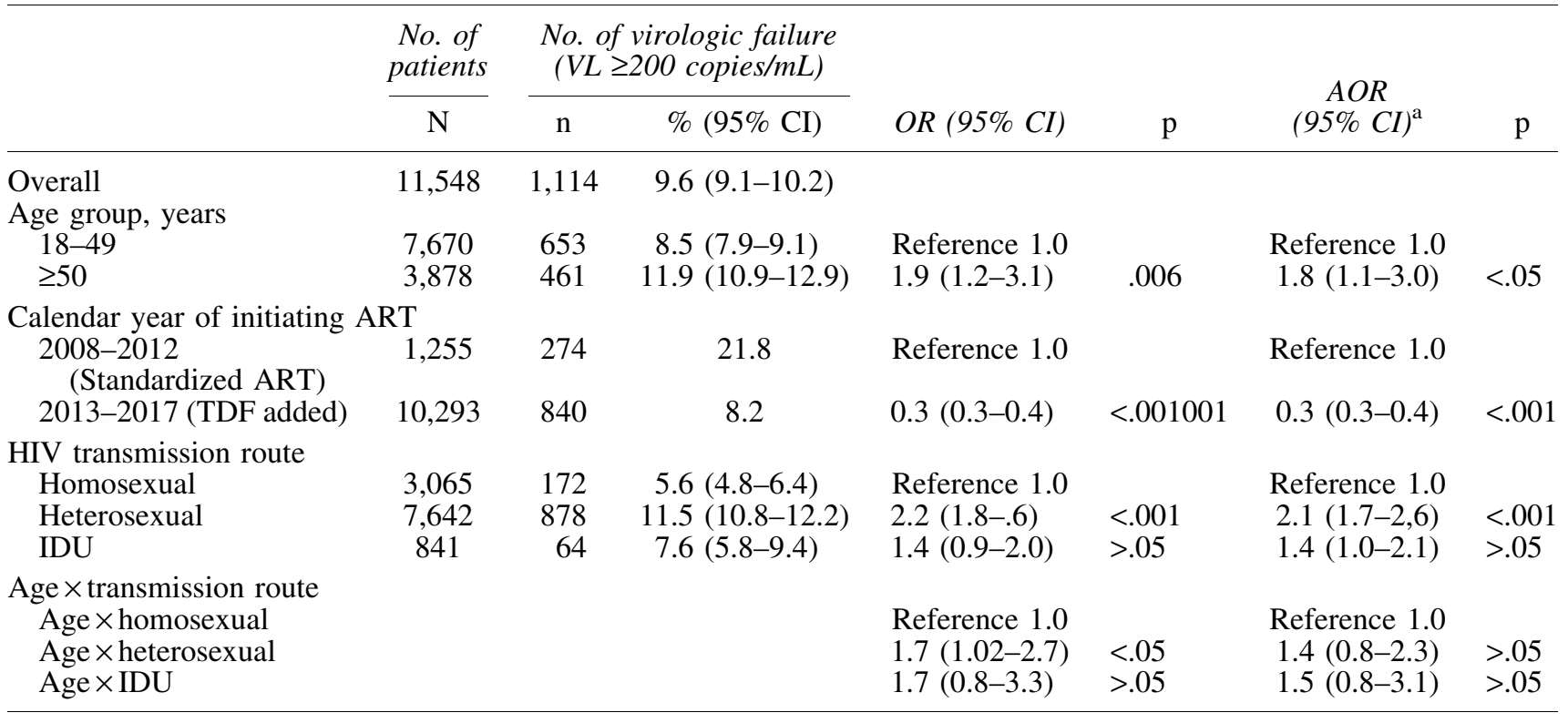

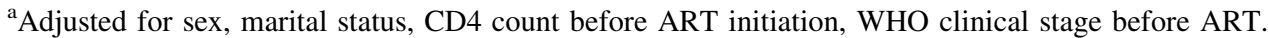

AOR, adjusted odds ratio; CI, confidence interval; IDU, injection drug use; OR, odds ratio; VL, viral load.

of initiating ART, HIV transmission route, and interaction term of age group by transmission route (Table 4).

\section{Discussion}

Our study showed that older PLWH had higher level of virologic failure and mortality within the first year of initiating ART than younger people. Later diagnosis of HIV infection may contribute to poorer disease outcomes among older people, as shown by lower CD4 count at HIV diagnosis in this age group. These findings are consistent with study findings in other countries. ${ }^{6,14-16}$ Another reason may be the delay in ART initiation: older people started ART in about half year of HIV diagnosis, which is half as 12 months among younger people. Older people may also have worse responses to HIV treatment, and this should be evaluated in future research.

Table 4. Comparison of Mortality Rate Between Age Groups of 18-49 and $\geq 50$ Years Within 12 Months of Initiating Antiretroviral Therapy in Chongqing, China, 2008-2017

\begin{tabular}{|c|c|c|c|c|c|c|c|c|}
\hline & $\begin{array}{c}\text { No. of } \\
\text { patients }\end{array}$ & $\begin{array}{l}\text { No. of } \\
\text { deaths }\end{array}$ & $\begin{array}{l}\text { Person } \\
\text { years }\end{array}$ & $\begin{array}{c}\text { Deaths per } \\
100 \text { person } \\
\text { years }(95 \% \text { CI) }\end{array}$ & $H R(95 \% C I)$ & $\mathrm{p}$ & $\begin{array}{c}A H R \\
(95 \% C I)^{\mathrm{a}}\end{array}$ & $\mathrm{P}$ \\
\hline $\begin{array}{l}\text { Overall } \\
\text { Age groun vears }\end{array}$ & 29,115 & 2,694 & 27,851 & $9.7(9.3-10.0)$ & & & & \\
\hline $\begin{array}{l}18-49 \\
\geq 50\end{array}$ & $\begin{array}{l}18,254 \\
10,861\end{array}$ & $\begin{array}{l}1,190 \\
1,504\end{array}$ & $\begin{array}{l}17,601 \\
10,250\end{array}$ & $\begin{array}{c}6.8(6.4-7.1) \\
14.7(14.0-15.4)\end{array}$ & $\begin{array}{l}\text { Reference 1.0 } \\
3.8(2.6-5.7)\end{array}$ & $<.001$ & $\begin{array}{l}\text { Reference } 1.0 \\
3.1(2.1-4.6)\end{array}$ & $<0.001$ \\
\hline $\begin{array}{l}\text { Calendar year of initiating } \\
\text { 2008-2012 } \\
\text { (Standardized ART) }\end{array}$ & $\begin{array}{l}\text { ART } \\
4,899\end{array}$ & 916 & $4,622.6$ & $19.8(18.7-21.0)$ & Reference 1.0 & & Reference 1.0 & \\
\hline 2013-2017 (TDF added) & 24,216 & 1,778 & $23,228.4$ & $7.7(7.3-8.0)$ & $0.4(0.4-0.4)$ & $<.001$ & $0.4(0.4-0.5)$ & $<0.001$ \\
\hline $\begin{array}{l}\text { HIV transmission route } \\
\text { Homosexual } \\
\text { Heterosexual } \\
\text { IDU }\end{array}$ & $\begin{array}{l}6,009 \\
21,574 \\
1,532\end{array}$ & $\begin{array}{c}139 \\
2,267 \\
288\end{array}$ & $\begin{array}{l}59,201 \\
20,549 \\
1,382\end{array}$ & $\begin{aligned} 2.3(2.0-2.7) \\
11.0(10.6-11.5) \\
20.8(18.7-23.0)\end{aligned}$ & $\begin{array}{l}\text { Reference } 1.0 \\
3.9(3.2-4.8) \\
11.1(8.9-13.9)\end{array}$ & $\begin{array}{l}<.001 \\
<.001\end{array}$ & $\begin{array}{l}\text { Reference } 1.0 \\
3.7(3.0-4.5) \\
7.7(6.1-9.7)\end{array}$ & $\begin{array}{l}<0.001 \\
<0.001\end{array}$ \\
\hline $\begin{array}{l}\text { Age } \times \text { transmission route } \\
\text { Age } \times \text { homosexual } \\
\text { Age } \times \text { heterosexual } \\
\text { Age } \times \text { IDU }\end{array}$ & & & & & $\begin{array}{l}\text { Reference } 1.0 \\
1.9(1.3-2.9) \\
3.8(2.1-6.8)\end{array}$ & $\begin{array}{r}.001 \\
<.001\end{array}$ & $\begin{array}{l}\text { Reference } 1.0 \\
1.5(1.0-2.3) \\
2.4(1.3-4.4)\end{array}$ & $\begin{array}{l}<0.054 \\
<0.01\end{array}$ \\
\hline
\end{tabular}

${ }^{\text {a} A d j u s t e d ~ f o r ~ s e x, ~ m a r i t a l ~ s t a t u s, ~ C D 4 ~ c o u n t ~ b e f o r e ~ A R T ~ i n i t i a t i o n, ~ W H O ~ c l i n i c a l ~ s t a g e ~ b e f o r e ~ A R T . ~}$

AHR, adjusted hazard ratio; HR, hazard ratio. 
The proportion of older people among all reported adult cases in Chongqing City has been increasing over years; it was greater than $50 \%$ in both male and female cases in 2017. It is suggested that HIV prevention and treatment programs should set priorities for older PLWH. Lower education and risk awareness levels among older PLWH may post challenges for effective implementation of HIV prevention and care. ${ }^{5}$

Compared with younger adult cases, older PLWH in Chongqing were more likely to be divorced and had heterosexual transmission. These profiles of HIV epidemic in Chongqing reflect the epidemic across China. An increased number of HIV infections has been reported among youths nationwide in recent years, particularly among young men who have sex with men.

The study has limitations. First, disease progression, including virologic failure and mortality was assessed within 12 months of ART initiation. The longer progression is unknown and should be further studied. Second, we used all-cause mortality within 12 months of ART initiation as a variable of measuring age-specific effect of ART treatment. The older age group might have more non-AIDS-related deaths than the younger group, and this may lead to biased estimation of the age-specific effect of HIV treatment. However, this effect of non-AIDS-related death is unlikely to be significant, as we measured the mortality in a short time period. Third, due to limited available information in public health databases, other factors such as ART regimens and ART compliance, which may influence disease outcomes, were not assessed in this study. A prospective cohort study design for assessing potential confounding factors could overcome this limitation.

Despite these limitations, this is the first study of evaluating HIV treatment outcomes among older PLWH in China. It provides valuable information on demographic and behavioral profiles, access to HIV care, and disease progression among older PLWH. As this study included a large sample enrolled over a span of three decades and there are similarities in HIV epidemic profiles and HIV care between Chongqing and China, including the same HIV testing and ART guidelines implemented across the country, increasing proportion of older people, high proportion of heterosexual transmission among older PLWH, the study results may be generalizable to HIVinfected populations in and beyond China. As over half of newly reported HIV/AIDS in 2017 were older people in Chongqing, it poses urgency and challenges for the HIV prevention and treatment programs in Chongqing and other Chinese cities to provide high-quality service to older patients.

\section{Author Disclosure Statement}

No competing financial interests exist.

\section{Funding Information}

This study was supported by grants from Key Medical Research Project of Chongqing Health and Family Planning Commission (2017ZDXM001), and from the "Neil Shen's SJTU Medical Research Fund."

\section{References}

1. UNAIDS: The Gap Report: People Aged 50 Years or Older. UNAIDS, Geneva, 2014.

2. Mahy M, Autenrieth CS, Stanecki K, Wynd S: Increasing trends in HIV prevalence among people aged 50 years and older: Evidence from estimates and survey data. AIDS 2014;28:S453.

3. Negin J, Cumming RG: HIV infection in older adults in sub-Saharan Africa: Extrapolating prevalence from existing data. Bull World Health Organ 2010;88:847853.

4. Tavoschi L, Dias JG, Pharris A, et al.: New HIV diagnoses among adults aged 50 years or older in 31 European countries, 2004-2015: An analysis of surveillance data. Lancet HIV 2017;4:e514-e521.

5. Chen $\mathrm{H}, \mathrm{Wu} \mathrm{X}$, Chen L, et al.: Rapidly spreading human immunodeficiency virus epidemic among older males and associated factors: A large-scale prospective cohort study in rural Southwest China. Sex Transm Dis 2019;46:234239.

6. Asher I, Guri KM, Elbirt D, et al:: Characteristics and outcome of patients diagnosed with HIV at older age. Medicine 2016;95(1):e2327.

7. Zingmond DS, Kilbourne AM, Justice AC, et al.: Differences in symptom expression in older HIV-positive patients: The Veterans Aging Cohort 3 Site Study and HIV Cost and Service Utilization Study experience. J Acquir Immune Defic Syndr 2003;33(Suppl 2):S84S92.

8. Parikh SM, Obuku EA, Walker SA, et al:: Clinical differences between younger and older adults with HIV/AIDS starting antiretroviral therapy in Uganda and Zimbabwe: A secondary analysis of the DART trial. PLoS One 2013;8: e76158.

9. Carriquiry G, Giganti MJ, Castilho JL, et al.: Virologic failure and mortality in older ART initiators in a multisite Latin American and Caribbean Cohort. J Int AIDS Soc 2018;21:e25088.

10. Eduardo E, Lamb MR, Kandula S, et al.: Characteristics and outcomes among older HIV-positive adults enrolled in HIV programs in four sub-Saharan African countries. PLoS One 2014;9:e103864.

11. Pilowsky DJ, Wu L-T: Sexual risk behaviors and HIV risk among Americans aged 50 years or older: A review. Subst Abuse Rehabil 2015;6:51.

12. Levy JA, Ory MG, Crystal S: HIV/AIDS interventions for midlife and older adults: Current status and challenges. J Acquir Immune Defic Syndr 2003;33:S59-S67.

13. Loeb DF, Lee RS, Binswanger IA, Ellison MC, Aagaard EM: Patient, resident physician, and visit factors associated with documentation of sexual history in the outpatient setting. J Gen Intern Med 2011;26:887-893.

14. Akers AY, Bernstein L, Doyle J, Corbie-Smith G: Older women and HIV testing: Examining the relationship between HIV testing history, age, and lifetime HIV risk behaviors. Sex Transm Dis 2008;35:420-423.

15. Smith RD, Delpech VC, Brown AE, Rice BD: HIV transmission and high rates of late diagnoses among adults aged 50 years and over. AIDS 2010;24:2109-2115.

16. Gullón A, Verdejo J, de Miguel R, Gómez A, Sanz J: Factors associated with late diagnosis of HIV infection and missed opportunities for earlier testing. AIDS Care 2016; 28:1296-1300. 
17. Luther VP, Wilkin AM: HIV infection in older adults. Clin Geriatr Med 2007;23:567-583.

18. Liu H, He X, Levy JA, Xu Y, Zang C, Lin X: Psychological impacts among older and younger people living with HIV/AIDS in Nanning, China. J Aging Res 2014;2014: 576592.

19. Li N, Li H, Ma YM, Fan PY, Yang WJ, Zhu Q: A qualitative study on high risk behaviors and related factors of reported HIV/AIDS cases aged 60 years and above in some areas of Henan province [in Chinese]. Zhonghua Liu Xing Bing Xue Za Zhi 2017;38:1161-1164.

20. Zhang Y, Xiao Q, Zhou L, et al.: The AIDS epidemic and economic input impact factors in Chongqing, China, from 2006 to 2012: A spatial-temporal analysis. BMJ Open 2015;5:e006669.

21. Mao Y, Wu Z, Poundstone K, et al.: Development of a unified web-based national HIV/AIDS information system in China. Int J Epidemiol 2010;39(Suppl 2):ii79-ii89.

22. High KP, Brennan-Ing M, Clifford DB, et al.: HIV and aging: State of knowledge and areas of critical need for research. A report to the NIH Office of AIDS Research by the HIV and Aging Working Group. J Acquir Immune Defic Syndr 2012;60(Suppl 1):S1-S18.
Address correspondence to:

Han-Zhu Qian

Shanghai Jiao Tong University-Yale Joint Center for Biostatistics and Data Science Biopharmacy Building Room 4A-225 800 Dongchuan Road, Minhang District

Shanghai 200240

China

E-mail: han-zhu.qian@yale.edu

Yuhua Ruan

State Key Laboratory of Infectious Disease Prevention and Control (SKLID) Chinese Center for Disease Control and Prevention (China CDC)

Collaborative Innovation Center for Diagnosis and Treatment of Infectious Diseases

Beijing 102206

China

E-mail: ruanyuhua92@163.com 